\title{
Retraction of: Redox-Sensing Iron-Sulfur Cluster Regulators, by Crack JC, Le Brun NE. Antioxid Redox Signal [Epub ahead of print]; DOI: 10.1089/ars.2017.7369, 2017.
}

\footnotetext{
The instant online e-pub version of the article entitled, Redox-Sensing Iron-Sulfur Cluster Regulators, by Crack JC and Le Brun NE, utilizing the DOI number 10.1089/ars.2017.7369 is being officially retracted from Antioxidants and Redox Signaling as it is an accidental duplicate submission by the authors to the Journal's peer review system.
} 